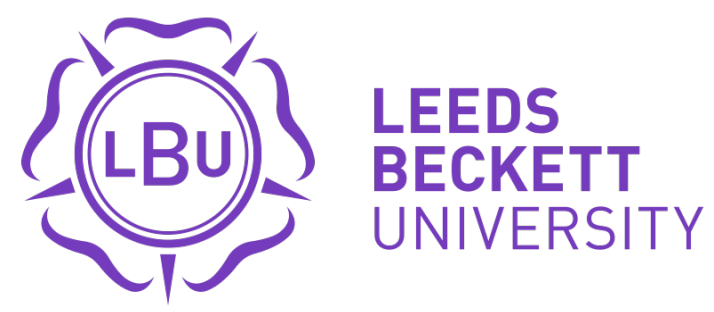

Citation:

Ramachandran, M and Sangaiah, AK and Goa, X-Z and Zheng, X (2015) A fuzzy DEMATEL approach based on intuitionistic fuzzy information for evaluating knowledge transfer effectiveness in GSD projects. International Journal of Innovative Computing and Applications, 6 (3-4). ISSN 1751-6498 DOI: https://doi.org/10.1504/IJICA.2015.073006

Link to Leeds Beckett Repository record:

https://eprints.leedsbeckett.ac.uk/id/eprint/1992/

Document Version:

Article (Submitted Version)

The aim of the Leeds Beckett Repository is to provide open access to our research, as required by funder policies and permitted by publishers and copyright law.

The Leeds Beckett repository holds a wide range of publications, each of which has been checked for copyright and the relevant embargo period has been applied by the Research Services team.

We operate on a standard take-down policy. If you are the author or publisher of an output and you would like it removed from the repository, please contact us and we will investigate on a case-by-case basis.

Each thesis in the repository has been cleared where necessary by the author for third party copyright. If you would like a thesis to be removed from the repository or believe there is an issue with copyright, please contact us on openaccess@leedsbeckett.ac.uk and we will investigate on a case-by-case basis. 


\title{
A Fuzzy DEMATEL Approach based on Intuitionistic Fuzzy Information for Evaluating Knowledge Transfer Effectiveness in GSD Projects
}

\author{
Arun Kumar Sangaiah', Xiao Zhi Gao ${ }^{2}$, Muthu Ramachandran ${ }^{3}$ \\ 1,VIT University, Vellone, Tamil Nadu, India \\ 2Aalto University, Aalto, Finland \\ 3Leeds Metropolitan University, United Kingdom \\ sarunkumar@ vit.ac.in ${ }^{1}$
}

\begin{abstract}
The offshore/onsite teams' effectiveness of knowledge transfer is significantly measured by a various kinds of factors. In this paper, we propose a Knowledge Transfer (KT) assessment framework which is integrates four criterions: knowledge, team, technology, and organization factors to evaluate KT effectiveness of GSD teams. In this context, we presents fuzzy DEMATEL approach for assessing GSD teams KT effectiveness based on intuitionistic fuzzy numbers (IFNs). In this approach, decision makers provided their subjective judgments on the criteria, characterized on the basis of intuitionistic fuzzy sets. Moreover, intuitionistic fuzzy sets are used in the fuzzy DEMATEL approach can be effectively assessing the KT effectiveness criteria, rank the alternatives. Subsequently, the complete process is illustrated with GSD teams' KT evaluation criteria samples, the factors are ranked using fuzzy linguistic variables which are mapped to IFNs. In that case, IFNs has been converted into their corresponding basic probability assignments (BPAs) and then Dempster-Shafer theory is used to combine the group decision making process. Besides, illustrate applicability and usefulness of proposed approach in group decision making process for evaluation of multiple criteria's under fuzzy environment has been tested by software professionals at Inowits software organization in India.
\end{abstract}

Keywords: Knowledge Transfer (KT), Global Software Development (GSD), intuitionistic fuzzy sets, Fuzzy DEMATEL, Basic Probability Assignments, Dempster-Shafer theory.

\section{Introduction}

In today's world, offshore/on-site teams' KT effectiveness has been one of the key determinants for evaluating GSD project outcome in the software service outsourcing context. GSD is primarily an outsourcing technique in which on-site teams' work involved in the client location, understanding and auditing the client requirements, whereas, offshore teams operating at 
different regions, execute the requirements based on the inputs provided by the on-site teams [1]. Since, many of our related works $[1,2,3,4,5,6,7,18]$ have emphasized the impact of knowledge transfer success in the Information System (IS) outsourcing. Consistent with earlier studies, this paper investigated the offshore/onsite teams KT effectiveness in GSD projects. Consequently, a comprehensive framework for considering GSD teams' knowledge, team, technology, and organization factors for assessing KT effectiveness in GSD projects has not been reported in the literature. Moreover, the number of studies $[8,13,14,16]$ has adopted IFNs for multi-criteria decision making (MCDM) problems. In addition, fuzzy group decision making based on IFNs for evaluating KT effectiveness in GSD projects has been very limited in the literature. Thus, addressing this research gaps inspired us to employ a fuzzy DEMATEL approach based on intuitionistic fuzzy information has been presented in this paper. Subsequently, the proposed approach will helpful for decision makers to solve the uncertainty and subjective vagueness in MCDM problems.

In our prior research, we have investigated thirty-five evaluation criteria for effectiveness of knowledge transfer among GSD teams' on the basis of combined fuzzy DEMATEL and fuzzy MCDM approach [7]. Based on the results of our earlier study [7], above studied approaches (DEMATEL-FMCDM) and interview with experts we have identified the majority of ten factors perceived as significant for measuring KT effectiveness in GSD projects as listed in Table.1. Consistent with earlier works [6,7], to address the enhanced way for identifying KT effectiveness factors in GSD projects, we have adopted fuzzy DEMATEL approach with IFNs in a group decision making process. In our prior work [7], fuzzy DEMATEL approach has been addressed to evaluate the KT factors. Moreover, we have utilized triangular fuzzy numbers has converted in to crisp scores consisting of number of defuzzification steps. Since, the traditional methods in defuzzification of fuzzy numbers have time consuming and may lead to an error factors. To overcome this problem, the combination of BPA, Dempster-Shafer theory and fuzzy DEMATEL approach has been presented in this paper. Similarly, Dempster-Shafer theory associated with fuzzy DEMATEL approach has been addressed in recent studies $[8,10]$ to avoid the need for defuzzification process in fuzzy DEMTEL approach. Likewise, this study integrated the Dempster-Shafer theory and fuzzy DEMATEL method for evaluating KT effectiveness in GSD projects. 
In recent times, a number of studies [5,7,8,9] have adopted a fuzzy DEMATEL approach for evaluation of attributes, interrelationship among the criteria and especially handling the subjective judgments that are vague and imprecise. In this paper, the relative importance of ten KT effectiveness factors has been assessed by thirty experts with the use of linguistic assessments. Further, the linguistic values are represented by IFNs which are represented by their BPA values. Moreover, conversion of an IFN into BPA and fusing them saves the effort of defuzzifying the value set. In addition, when a large set of values are concerned, traditional ways of defuzzification approach becoming tedious and time consuming. As a result, Dempster-Shafer theory has been presented in this study to avoid the need of defuzzification and to fuse the BPA, and then fuzzy DEMATEL approach can be applied on each BPA. Consequently, the very few studies $[8,10]$ have integrated that Dempster-Shafer theory and fuzzy DEMATEL method for group decision making process. Thus, addressing this research gaps inspired us to propose a combined approach based on fuzzy DEMATEL- Dempster-Shafer theory for evaluating KT effectiveness in GSD projects presented in this paper.

\begin{tabular}{|c|c|c|}
\hline Factors & Context & Description \\
\hline F1 & Knowledge & Gathering the information and experiences among teams. \\
\hline F2 & Knowledge & Sharing the knowledge and experiences among team members \\
\hline F3 & Knowledge & Knowledge incentive towards business process and project outcome \\
\hline F4 & Organization & Specialty and knowledge creation ability among teams. \\
\hline F5 & Knowledge & Team members ability to provide assistance to solving problems \\
\hline F6 & Team & Personal attachment and support towards the project. \\
\hline F7 & Team & Co-ordination of teams to achieve the project goals. \\
\hline F8 & Knowledge & $\begin{array}{l}\text { Learning and sharing the technical information and work materials } \\
\text { of teams }\end{array}$ \\
\hline F9 & Technology & $\begin{array}{c}\text { Explicit and standard communication pattern for knowledge transfer } \\
\text { effectiveness. }\end{array}$ \\
\hline F10 & Technology & Tools and technology to facilitate knowledge transfer within teams. \\
\hline
\end{tabular}

To address these research gaps, an empirical study has been carried out in Indian software companies to evaluate KT effectiveness of the GSD teams. The rest of this paper is organized as follows: Section 2 presents the theoretical foundations of Dempster-Shafer theory and fuzzy DEMATEL approach. Section 3 and 4 presents the empirical study and assessment framework 
used in this research respectively. Section 5 and section 6 presents the findings of the study and concluding remarks of the study has presented to address the significance of KT effectiveness on GSD teams.

\section{Theoretical Foundations}

In this study, the fuzzy DEMATEL approach has been integrated with the Dempster-Shafer theory for evaluation of KT effectiveness from the perspective of GSD teams' via IFNs is proposed. The proposed methodologies for KT effectiveness on GSD project outcome evaluation framework consists of two aspects. First, the linguistic values are represented by IFNs which are represented by their BPA values before utilizing DEMATEL method. Finally, Dempster-Shafer theory has been utilized to avoid the need of defuzzification and to fuse the BPA, followed by fuzzy DEMATEL approach can be applied on each BPA. Despite the importance of defuzzification in fuzzy modeling and MCDM the number of earlier studies has utilized Dempster-Shafer in defuzzification process $[12,17]$. In Dempster-Shafer theory, the information from each source is seen as a piece of evidences which is represented by a basic probability assignment (BPA) [12]. This study processes the IFNs with help of BPAs in order to do away with a complex defuzzification process. The theoretical foundations of Dempster-Shafer theory and fuzzy DEMATEL have been illustrated in the following sections.

\subsection{Intuitionistic fuzzy sets (IFS)}

Intuitionistic fuzzy sets are sets whose elements have degrees of membership and nonmembership. Intuitionistic fuzzy sets have been introduced by Krassimir Atanassov [15]. The IFS is formally defined as follows:

Definition: For any fuzzy set (let's say) $X$ be fixed universe. Let $A$ be the subset of $X$. An IFS $A^{*}$ in $X$ construct set have the form $A^{*}=\left\{\left\langle x, \mu_{A}(x), \vartheta_{A}(x)\right\rangle: x \in E\right\}$ where $\mu_{A}: X \rightarrow[0,1]$ and $\vartheta_{A}: X \rightarrow[0,1]$ satisfy the condition $0 \leq \mu_{A}(x)+\vartheta_{A}(x) \leq 1$. Here, $A^{*}$ represents Intuitionistic fuzzy sets. The function $\mu_{A}: X \rightarrow[0,1]$ and $\vartheta_{A}: X \rightarrow[0,1]$ denotes degree of membership and non-membership respectively. 


\subsection{Dempster-Shafer theory}

Currently, there is an interest to use the evidential reasoning approach on the basis of DempsterShafer theory in MCDM analysis Moreover, the significance of Dempster-Shafer theory is greatly acknowledged in substantial measure of existing literature $[11,20]$. In addition, the earlier studies have incorporated Dempster-Shafer theory in MCDM for various kinds of applications $[8,10,12]$. Likewise, the Dempster-Shafer theory is incorporated for analyzing the data in this study, and its computational procedure which is summarized as follows:

Step 1: Set up intuitionistic fuzzy decision matrix

Create a decision matrix $\mathrm{A}$ for the important weights of KT effectiveness factors $\left(C_{j} j=\right.$ $1,2,3, \ldots, n)$. The respondents $\left(R^{i}, i=1,2,3, \ldots m\right)$ provided their subjective judgments about the importance weights of each KT factors by using linguistic scales (as listed in Table 2). The direct relation matrix $\mathrm{A}$ is as follows:

$$
\begin{aligned}
& \begin{array}{lllll}
R^{l} & R^{2} & R^{3} & \ldots & R^{m}
\end{array} \\
& C_{1}\left[\begin{array}{lllll}
\tilde{a}_{1}^{1} & \tilde{a}_{1}^{2} & \tilde{a}_{1}^{3} & \ldots & \tilde{a}_{1}^{m} \\
\tilde{a}_{2} & a_{2} & a_{2} & \ldots & a_{2}
\end{array}\right] \\
& C_{2} \mid \begin{array}{lllll}
\tilde{a}_{2}^{1} & \tilde{a}_{2}^{2} & \tilde{a}_{2}^{3} & \ldots & \tilde{a}_{2}^{m}
\end{array}
\end{aligned}
$$

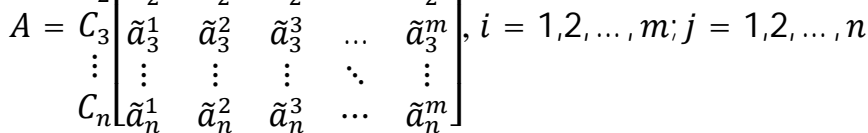

In this decision matrix $m, n$ denote the number of respondents and the number of criteria respectively. $\tilde{a}_{j}^{i}=\left(L a_{j}^{i} U a_{j}^{i}\right)$ represents Lower-bound (L), Upper bound (U) of a IFNs and fuzzy degree of impact as assessed by $\mathrm{i}^{\text {th }}$ respondents for $\mathrm{j}^{\text {th }}$ influential factor.

\begin{tabular}{|rc|}
\hline \multicolumn{1}{|c|}{ Linguistic Variable } & Corresponding IFNs \\
\hline Extremely unimportant & $(0.1,0.9)$ \\
\hline Unimportant & $(0.35,0.6)$ \\
\hline Either important or unimportant & $(0.5,0.45)$ \\
\hline Important & $(0.75,0.2)$ \\
\hline Extremely Important & $(0.9,0.1)$ \\
\hline
\end{tabular}

Table 2: Fuzzy membership with IFNs

Step 2: Design the fuzzy linguistic variables 
The study addresses uncertainty and subjective vagueness of human assessments dealt with fuzzy linguistic variable: Extremely unimportant, unimportant, either important or unimportant, important, Extremely Important and their corresponding IFNs are listed in Table-2.

\section{Step 3: Transform linguistic variables into IFN matrix.}

Convert the linguistic assessment into IFN matrices to express the relations. Each linguistic variable is represented by an IFNs of the format $\left(a_{1}, a_{2}\right)$ as shown in Table-2. The initial IFN matrices for three experts' group opinions are tabulated in Table-3 to Table-5.

\section{Step 4: Conversion IFNs to BPA.}

In this study, IFN matrices are converted into BPA matrixes. For example: if an IFN A is represented by $\left(a_{1}, a_{2}\right)$ where $a_{1}$ is the degree of membership $m(y)$ and $a_{2}$ is the degree of nonmembership $m(n)$, then the degree of uncertainty $m(\theta)$ would be calculated as $m(\theta)=1-$ $m(y)-m(n)$. The corresponding BPA matrices for $m(y), m(n)$, and $m(\theta)$ are represented in Table-6 to Table-11.

Step 5: Fuse BPAs by Dempster's rule of combination.

To apply Dempster's rule of combination in Eq.(1) to every element of the BPA matrixes, a comprehensive matrix which represents the initial relation can be constructed [8].

$m(A)=\frac{1}{1-K} \sum_{B \cap C=A} m_{1}(B) m_{2}(C)$

With

$K=\sum_{B \cap C=\varnothing} m_{1}(B) m_{2}(C)$

Where $\mathrm{K}$ is a normalization constant, applicable when $K<1$. The fused matrices for $m(y)$ and $m(n)$ are given in Table-12 and Table -13 .

Step 6: Apply fuzzy DEMATEL approach

Apply DEMATEL approach to calculate the overall relation with respect to the BPA of each proposition as shown Eqn.(3)-Eqn.(5). Then, the relative importance of factors can be identified from different aspects. 


\subsection{Fuzzy DEMATEL}

The DEMATEL method is incorporated for analyzing the data in this study, and its computational procedure which is summarized as follows:

\section{Step 1: Construct initial direct relational matrix $D$}

On performing defuzzification of the given values through Dempster-Shafer theory, we have obtained the initial direct-relation matrix D. Each element $d_{i j}$ is a positive integer that denotes the impact of criteria $i$ on factor $j$. It is important to note that the diagonal elements $d_{i j}$ are equal to 0 .

\section{Step 2: Obtain normalized decision matrix $N$}

Normalized matrix is $N=\left[n_{i j}\right]$ obtained using Eq. (3) and (4) as shown:

$$
\begin{aligned}
& m=\max _{1 \leq i \leq n} \sum_{j=1}^{n} d_{i j} \\
& N=\frac{1}{m} D
\end{aligned}
$$

Step 3: Compute the total relational matrix $F$

In order to calculate total relation matrix $\mathrm{F}$, an identity matrix has to be constructed of the same size as the normalized matrix $\mathrm{N}$. Total relation matrix F can be calculated using the formula given in Eq. (5)

$F=N(I-N)^{-1}$

Step 4: Obtain the sum of rows and columns to compute prominence and relation of each criterion

Identify most significant factors and least significant factors in widespread consideration of the indexes R-C on the BPA of each proposition. Considering the priority of each factor, the ones which are more significant and can greatly improve the efficiency of the KT effectiveness. 


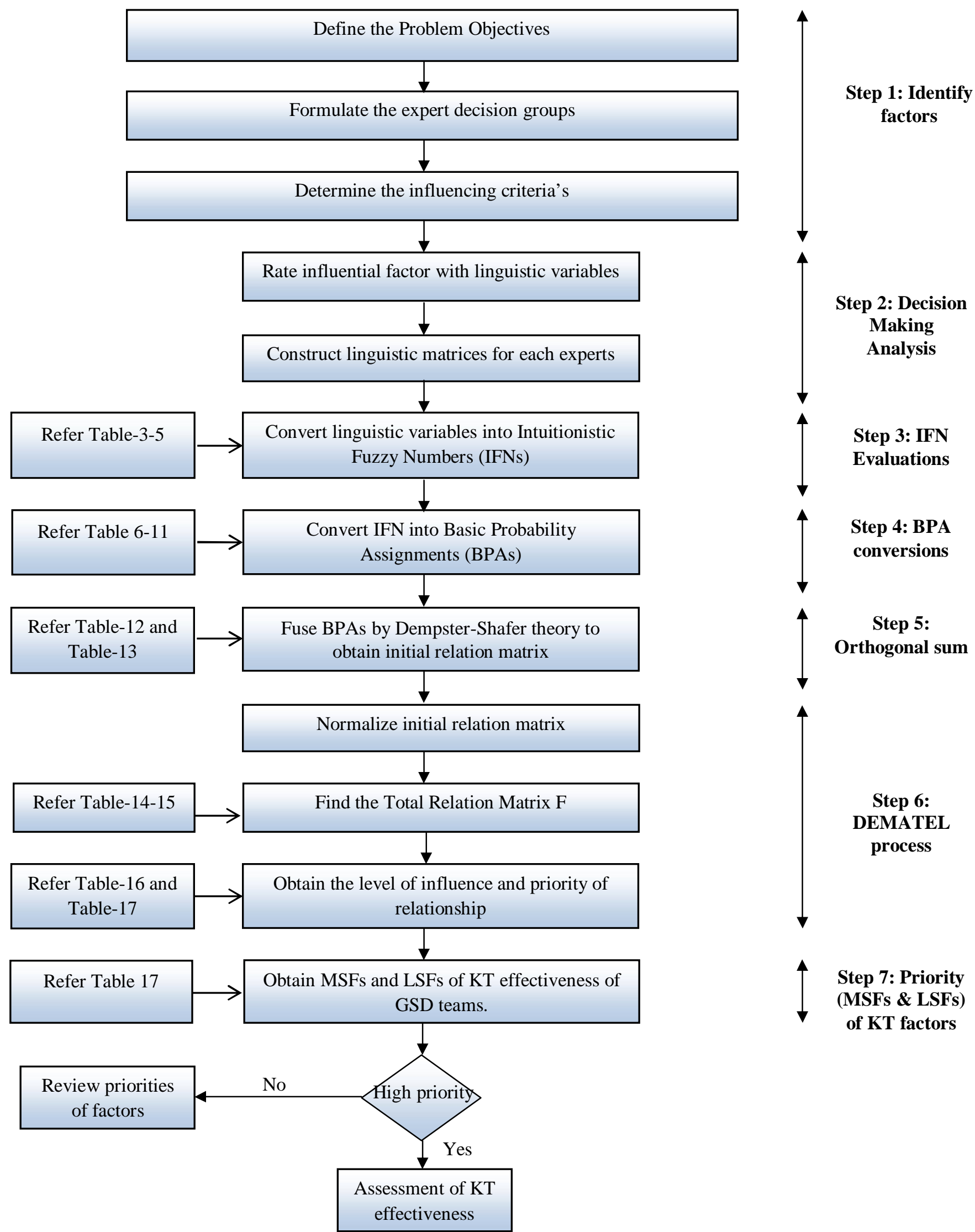

Figure.1: The flow diagram of the proposed Dempster Shafer theory - DEMATEL Framework for evaluating GSD teams KT effectiveness 


\section{Framework for evaluating KT effectiveness of GSD teams.}

To the best of our knowledge, up to date research on evaluating GSD teams KT effectiveness is very limited. Moreover, the assessment framework for the integration of knowledge, team characteristics, technology, and organizational elements for the effectiveness of KT perceived by GSD teams has not been adequately presented in the available literature. Further, the hybridization of Dempster-Shafer theory and fuzzy DEMATEL method for group decision making has addressed only in very few studies. Based on this context, we have integrated Dempster-Shafer theory and DEMATEL approach for evaluating GSD teams' KT effectiveness via intuitionistic fuzzy numbers (IFNs). The construction of proposed framework and its computation procedure under a fuzzy environment is depicted in Fig.1.

\section{Empirical case for evaluating the KT criteria's of GSD teams}

The primary objective of this study is to investigate the influence of GSD teams' KT effectiveness at individual and organization levels in organizational behavior (OB) research phenomenon of GSD projects. To achieve this goal, an empirical study has been carried out in Inowits software organization located in India. The organization has more than 100 employees, reputation (ISO certification) and provides offshore outsourcing service support to various countries. The demographic details of the respondents of this study were three expert decision groups (executive committee, solution development team, and solution delivery team) of this organization. Consequently, the empirical study has been tested among thirty experts of this organization to validate the effectiveness of KT factors (see table 1) of GSD teams. The hybrid Dempster-Shafer theory and DEMATEL approaches were applied in this case study, as illustrated in the following sections.

\subsection{Measurement of KT effectiveness criteria's by Dempster-Shafer theory}

The basic computational procedure of Dempster-Shafer theory used in this study is as follows:

Step 1: KT effectiveness criteria were identified through related literature and our earlier study [7]. Then, create a fuzzy decision matrix with respect to the subjective decisions of 30 experts 
use the linguistic assessments for the possible rating of ten KT effectiveness criteria and their corresponding IFNs as shown in Table-2.

\begin{tabular}{|ccccccccccc|}
\hline & F1 & F2 & F3 & F4 & F5 & F6 & F7 & F8 & F9 & F10 \\
\hline F1 & $(0.9,0.1)$ & $(0.9,0.1)$ & $(0.9,0.1)$ & $(0.9,0.1)$ & $(0.9,0.1)$ & $(0.9,0.1)$ & $(0.9,0.1)$ & $(0.9,0.1)$ & $(0.9,0.1)$ & $(0.9,0.1)$ \\
\hline F2 & $(0.75,0.2)$ & $(0.9,0.1)$ & $(0.9,0.1)$ & $(0.9,0.1)$ & $(0.9,0.1)$ & $(0.9,0.1)$ & $(0.9,0.1)$ & $(0.9,0.1)$ & $(0.9,0.1)$ & $(0.9,0.1)$ \\
\hline F3 & $(0.9,0.1)$ & $(0.9,0.1)$ & $(0.9,0.1)$ & $(0.9,0.1)$ & $(0.9,0.1)$ & $(0.9,0.1)$ & $(0.75,0.2)$ & $(0.9,0.1)$ & $(0.9,0.1)$ & $(0.9,0.1)$ \\
\hline F4 & $(0.9,0.1)$ & $(0.9,0.1)$ & $(0.9,0.1)$ & $(0.9,0.1)$ & $(0.9,0.1)$ & $(0.9,0.1)$ & $(0.5,0.45)$ & $(0.75,0.2)$ & $(0.75,0.2)$ & $(0.75,0.2)$ \\
\hline F5 & $(0.9,0.1)$ & $(0.9,0.1)$ & $(0.9,0.1)$ & $(0.9,0.1)$ & $(0.9,0.1)$ & $(0.9,0.1)$ & $(0.9,0.1)$ & $(0.9,0.1)$ & $(0.9,0.1)$ & $(0.9,0.1)$ \\
\hline F6 & $(0.9,0.1)$ & $(0.75,0.2)$ & $(0.75,0.2)$ & $(0.75,0.2)$ & $(0.75,0.2)$ & $(0.9,0.1)$ & $(0.9,0.1)$ & $(0.9,0.1)$ & $(0.9,0.1)$ & $(0.9,0.1)$ \\
\hline F7 & $(0.9,0.1)$ & $(0.75,0.2)$ & $(0.9,0.1)$ & $(0.75,0.2)$ & $(0.9,0.1)$ & $(0.9,0.1)$ & $(0.75,0.2)$ & $(0.9,0.1)$ & $(0.9,0.1)$ & $(0.9,0.1)$ \\
\hline F8 & $(0.75,0.2)$ & $(0.75,0.2)$ & $(0.9,0.1)$ & $(0.9,0.1)$ & $(0.75,0.2)$ & $(0.75,0.2)$ & $(0.9,0.1)$ & $(0.9,0.1)$ & $(0.9,0.1)$ & $(0.9,0.1)$ \\
\hline F9 & $(0.9,0.1)$ & $(0.75,0.2)$ & $(0.9,0.1)$ & $(0.9,0.1)$ & $(0.9,0.1)$ & $(0.9,0.1)$ & $(0.75,0.2)$ & $(0.9,0.1)$ & $(0.9,0.1)$ & $(0.9,0.1)$ \\
\hline F10 & $(0.75,0.2)$ & $(0.75,0.2)$ & $(0.75,0.2)$ & $(0.9,0.1)$ & $(0.75,0.2)$ & $(0.9,0.1)$ & $(0.9,0.1)$ & $(0.75,0.2)$ & $(0.9,0.1)$ & $(0.9,0.1)$ \\
\hline
\end{tabular}

Table 3: Initial direct relational matrix and corresponding IFNs of the expert 1

\begin{tabular}{|ccccccccccc|}
\hline & F1 & F2 & F3 & F4 & F5 & F6 & F7 & F8 & F9 & F10 \\
\hline F1 & $(0.75,0.2)$ & $(0.9,0.1)$ & $(0.9,0.1)$ & $(0.75,0.2)$ & $(0.9,0.1)$ & $(0.9,0.1)$ & $(0.75,0.2)$ & $(0.9,0.1)$ & $(0.75,0.2)$ & $(0.75,0.2)$ \\
\hline F2 & $(0.9,0.1)$ & $(0.9,0.1)$ & $(0.9,0.1)$ & $(0.75,0.2)$ & $(0.9,0.1)$ & $(0.75,0.2)$ & $(0.75,0.2)$ & $(0.9,0.1)$ & $(0.75,0.2)$ & $(0.75,0.2)$ \\
\hline F3 & $(0.75,0.2)$ & $(0.75,0.2)$ & $(0.75,0.2)$ & $(0.9,0.1)$ & $(0.9,0.1)$ & $(0.9,0.1)$ & $(0.75,0.2)$ & $(0.75,0.2)$ & $(0.75,0.2)$ & $(0.75,0.2)$ \\
\hline F4 & $(0.9,0.1)$ & $(0.9,0.1)$ & $(0.9,0.1)$ & $(0.9,0.1)$ & $(0.9,0.1)$ & $(0.5,0.45)$ & $(0.75,0.2)$ & $(0.75,0.2)$ & $(0.9,0.1)$ & $(0.9,0.1)$ \\
\hline F5 & $(0.9,0.1)$ & $(0.75,0.2)$ & $(0.75,0.2)$ & $(0.75,0.2)$ & $(0.9,0.1)$ & $(0.5,0.45)$ & $(0.75,0.2)$ & $(0.9,0.1)$ & $(0.9,0.1)$ & $(0.9,0.1)$ \\
\hline F6 & $(0.9,0.1)$ & $(0.9,0.1)$ & $(0.9,0.1)$ & $(0.75,0.2)$ & $(0.75,0.2)$ & $(0.9,0.1)$ & $(0.75,0.2)$ & $(0.75,0.2)$ & $(0.9,0.1)$ & $(0.9,0.1)$ \\
\hline F7 & $(0.9,0.1)$ & $(0.9,0.1)$ & $(0.9,0.1)$ & $(0.9,0.1)$ & $(0.9,0.1)$ & $(0.9,0.1)$ & $(0.9,0.1)$ & $(0.75,0.2)$ & $(0.5,0.45)$ & $(0.5,0.45)$ \\
\hline F8 & $(0.75,0.2)$ & $(0.75,0.2)$ & $(0.75,0.2)$ & $(0.9,0.1)$ & $(0.9,0.1)$ & $(0.75,0.2)$ & $(0.9,0.1)$ & $(0.9,0.1)$ & $(0.9,0.1)$ & $(0.9,0.1)$ \\
\hline F9 & $(0.9,0.1)$ & $(0.75,0.2)$ & $(0.75,0.2)$ & $(0.75,0.2)$ & $(0.75,0.2)$ & $(0.75,0.2)$ & $(0.75,0.2)$ & $(0.75,0.2)$ & $(0.9,0.1)$ & $(0.9,0.1)$ \\
\hline F10 & $(0.9,0.1)$ & $(0.9,0.1)$ & $(0.9,0.1)$ & $(0.9,0.1)$ & $(0.75,0.2)$ & $(0.75,0.2)$ & $(0.9,0.1)$ & $(0.9,0.1)$ & $(0.5,0.45)$ & $(0.5,0.45)$ \\
\hline
\end{tabular}

Table 4: Initial direct relational matrix and corresponding IFNs of the expert 2

\begin{tabular}{|c|c|c|c|c|c|c|c|c|c|c|}
\hline & F1 & F2 & F3 & F4 & F5 & F6 & F7 & F8 & F9 & F10 \\
\hline F1 & $(0.75,0.2)$ & $(0.9,0.1)$ & $(0.9,0.1)$ & $(0.75,0.2)$ & $(0.75,0.2)$ & $(0.75,0.2)$ & $(0.75,0.2)$ & $(0.50,0.45)$ & $(0.75,0.2)$ & $(0.75,0.2)$ \\
\hline F2 & $(0.9,0.1)$ & $(0.9,0.1)$ & $(0.9,0.1)$ & $(0.9,0.1)$ & $(0.75,0.2)$ & $(0.75,0.2)$ & $(0.50,0.45)$ & $(0.75,0.2)$ & $(0.75,0.2)$ & $(0.75,0.2)$ \\
\hline F3 & $(0.75,0.2)$ & $(0.35,0.6)$ & $(0.35,0.6)$ & $(0.9,0.1)$ & $(0.9,0.1)$ & $(0.9,0.1)$ & $(0.75,0.2)$ & $(0.1,0.9)$ & $(0.9,0.1)$ & $(0.9,0.1)$ \\
\hline F4 & $(0.75,0.2)$ & $(0.75,0.2)$ & $(0.75,0.2)$ & $(0.75,0.2)$ & $(0.50,0.45)$ & $(0.9,0.1)$ & $(0.75,0.2)$ & $(0.75,0.2)$ & $(0.75,0.2)$ & $(0.75,0.2)$ \\
\hline F5 & $(0.75,0.2)$ & $(0.75,0.2)$ & $(0.75,0.2)$ & $(0.35,0.6)$ & $(0.50,0.45)$ & $(0.75,0.2)$ & $(0.9,0.1)$ & $(0.75,0.2)$ & $(0.50,0.45)$ & $(0.50,0.45)$ \\
\hline F6 & $(0.9,0.1)$ & $(0.75,0.2)$ & $(0.75,0.2)$ & $(0.75,0.2)$ & $(0.75,0.2)$ & $(0.50,0.45)$ & $(0.50,0.45)$ & $(0.75,0.2)$ & $(0.75,0.2)$ & $(0.75,0.2)$ \\
\hline F7 & $(0.9,0.1)$ & $(0.9,0.1)$ & $(0.9,0.1)$ & $(0.75,0.2)$ & $(0.75,0.2)$ & $(0.75,0.2)$ & $(0.75,0.2)$ & $(0.50,0.45)$ & $(0.50,0.45)$ & $(0.50,0.45)$ \\
\hline F8 & $(0.50,0.45)$ & $(0.75,0.2)$ & $(0.75,0.2)$ & $(0.75,0.2)$ & $(0.75,0.2)$ & $(0.50,0.45)$ & $(0.50,0.45)$ & $(0.50,0.45)$ & $(0.75,0.2)$ & $(0.75,0.2)$ \\
\hline F9 & $(0.75,0.2)$ & $(0.50,0.45)$ & $(0.50,0.45)$ & $(0.50,0.45)$ & $(0.75,0.2)$ & $(0.9,0.1)$ & $(0.75,0.2)$ & $(0.35,0.6)$ & $(0.35,0.6)$ & $(0.35,0.6)$ \\
\hline F10 & $(0.9,0.1)$ & $(0.75,0.2)$ & $(0.75,0.2)$ & $(0.9,0.1)$ & $(0.50,0.45)$ & $(0.9,0.1)$ & $(0.75,0.2)$ & $(0.75,0.2)$ & $(0.9,0.1)$ & $(0.9,0.1)$ \\
\hline
\end{tabular}

Table 5: Initial direct relational matrix and corresponding IFNs of the expert 3

Step 2: Design the fuzzy linguistic variables. Convert the fuzzy linguistic variables into IFNs. The initial direct relational matrixes to express the relations on KT criteria and their corresponding IFNs of the experts as shown in Table-3 to Table-5 
Step 3: For integrating experts' subjective decisions via IFN matrixes effectively, then IFN matrixes are converted to BPA matrixes as shown in Table-6 to Table-11.

\begin{tabular}{|c|c|c|c|c|c|c|c|c|c|c|c|c|c|c|c|}
\hline & \multicolumn{4}{|c|}{ F1 } & \multicolumn{3}{|c|}{ F2 } & \multicolumn{3}{|c|}{ F3 } & \multicolumn{4}{c|}{ F4 } & \multicolumn{3}{c|}{ F5 } \\
\hline & $m(y)$ & $m(n)$ & $m(\theta)$ & $m(y)$ & $m(n)$ & $m(\theta)$ & $m(y)$ & $m(n)$ & $m(\theta)$ & $m(y)$ & $m(n)$ & $m(\theta)$ & $m(y)$ & $m(n)$ & $m(\theta)$ \\
\hline F1 & 0.9 & 0.1 & 0 & 0.9 & 0.1 & 0 & 0.9 & 0.1 & 0 & 0.9 & 0.1 & 0 & 0.9 & 0.1 & 0 \\
\hline F2 & 0.75 & 0.2 & 0.05 & 0.9 & 0.1 & 0 & 0.9 & 0.1 & 0 & 0.9 & 0.1 & 0 & 0.9 & 0.1 & 0 \\
\hline F3 & 0.9 & 0.1 & 0 & 0.9 & 0.1 & 0 & 0.9 & 0.1 & 0 & 0.9 & 0.1 & 0 & 0.9 & 0.1 & 0 \\
\hline F4 & 0.9 & 0.1 & 0 & 0.9 & 0.1 & 0 & 0.9 & 0.1 & 0 & 0.9 & 0.1 & 0 & 0.9 & 0.1 & 0 \\
\hline F5 & 0.9 & 0.1 & 0 & 0.9 & 0.1 & 0 & 0.9 & 0.1 & 0 & 0.9 & 0.1 & 0 & 0.9 & 0.1 & 0 \\
\hline F6 & 0.9 & 0.1 & 0 & 0.75 & 0.2 & 0.05 & 0.75 & 0.2 & 0.05 & 0.75 & 0.2 & 0.05 & 0.75 & 0.2 & 0.05 \\
\hline F7 & 0.9 & 0.1 & 0 & 0.75 & 0.2 & 0.05 & 0.9 & 0.1 & 0 & 0.75 & 0.2 & 0.05 & 0.9 & 0.1 & 0 \\
\hline F8 & 0.75 & 0.2 & 0.05 & 0.75 & 0.2 & 0.05 & 0.9 & 0.1 & 0 & 0.9 & 0.1 & 0 & 0.75 & 0.2 & 0.05 \\
\hline F9 & 0.9 & 0.1 & 0 & 0.75 & 0.2 & 0.05 & 0.9 & 0.1 & 0 & 0.9 & 0.1 & 0 & 0.9 & 0.1 & 0 \\
\hline F10 & 0.75 & 0.2 & 0.05 & 0.75 & 0.2 & 0.05 & 0.75 & 0.2 & 0.05 & 0.9 & 0.1 & 0 & 0.75 & 0.2 & 0.05 \\
\hline
\end{tabular}

\section{Table 6: The value of BPA for the expert group-1}

\begin{tabular}{|c|c|c|c|c|c|c|c|c|c|c|c|c|c|c|c|}
\hline & \multicolumn{4}{|c|}{ F6 } & \multicolumn{3}{|c|}{ F7 } & \multicolumn{3}{c|}{ F8 } & \multicolumn{3}{|c|}{ F9 } & \multicolumn{2}{c|}{ F10 } \\
\hline & $m(y)$ & $m(n)$ & $m(\theta)$ & $m(y)$ & $m(n)$ & $m(\theta)$ & $m(y)$ & $m(n)$ & $m(\theta)$ & $m(y)$ & $m(n)$ & $m(\theta)$ & $m(y)$ & $m(n)$ & $m(\theta)$ \\
\hline F1 & 0.9 & 0.1 & 0 & 0.9 & 0.1 & 0 & 0.9 & 0.1 & 0 & 0.9 & 0.1 & 0 & 0.9 & 0.1 & 0.00 \\
\hline F2 & 0.9 & 0.1 & 0 & 0.9 & 0.1 & 0 & 0.9 & 0.1 & 0 & 0.9 & 0.1 & 0 & 0.9 & 0.1 & 0.00 \\
\hline F3 & 0.9 & 0.1 & 0 & 0.75 & 0.2 & 0.05 & 0.9 & 0.1 & 0 & 0.9 & 0.1 & 0 & 0.9 & 0.1 & 0.00 \\
\hline F4 & 0.9 & 0.1 & 0 & 0.50 & 0.45 & 0.05 & 0.75 & 0.2 & 0.05 & 0.75 & 0.2 & 0.05 & 0.75 & 0.2 & 0.05 \\
\hline F5 & 0.9 & 0.1 & 0 & 0.9 & 0.1 & 0 & 0.9 & 0.1 & 0 & 0.9 & 0.1 & 0 & 0.9 & 0.1 & 0.00 \\
\hline F6 & 0.9 & 0.1 & 0 & 0.9 & 0.1 & 0 & 0.9 & 0.1 & 0 & 0.9 & 0.1 & 0 & 0.9 & 0.1 & 0.00 \\
\hline F7 & 0.9 & 0.1 & 0 & 0.75 & 0.2 & 0.05 & 0.9 & 0.1 & 0 & 0.9 & 0.1 & 0 & 0.9 & 0.1 & 0.00 \\
\hline F8 & 0.75 & 0.2 & 0.05 & 0.9 & 0.1 & 0 & 0.9 & 0.1 & 0 & 0.9 & 0.1 & 0 & 0.9 & 0.1 & 0.00 \\
\hline F9 & 0.9 & 0.1 & 0 & 0.75 & 0.2 & 0.05 & 0.9 & 0.1 & 0 & 0.9 & 0.1 & 0 & 0.9 & 0.1 & 0.00 \\
\hline F10 & 0.9 & 0.1 & 0 & 0.9 & 0.1 & 0 & 0.75 & 0.2 & 0.05 & 0.9 & 0.1 & 0 & 0.9 & 0.1 & 0.00 \\
\hline
\end{tabular}

Table 7: The value of BPA for the expert group-1 (contd.)

\begin{tabular}{|c|c|c|c|c|c|c|c|c|c|c|c|c|c|c|c|}
\hline & \multicolumn{4}{|c|}{ F1 } & \multicolumn{3}{|c|}{ F2 } & \multicolumn{3}{|c|}{ F3 } & \multicolumn{3}{|c|}{ F4 } & \multicolumn{2}{|c|}{ F5 } \\
\hline & $m(y)$ & $m(n)$ & $m(\theta)$ & $m(y)$ & $m(n)$ & $m(\theta)$ & $m(y)$ & $m(n)$ & $m(\theta)$ & $m(y)$ & $m(n)$ & $m(\theta)$ & $m(y)$ & $m(n)$ & $m(\theta)$ \\
\hline F1 & 0.75 & 0.2 & 0.05 & 0.9 & 0.1 & 0 & 0.9 & 0.1 & 0 & 0.75 & 0.2 & 0.05 & 0.9 & 0.1 & 0 \\
\hline F2 & 0.9 & 0.1 & 0 & 0.9 & 0.1 & 0 & 0.9 & 0.1 & 0 & 0.75 & 0.2 & 0.05 & 0.9 & 0.1 & 0 \\
\hline F3 & 0.75 & 0.2 & 0.05 & 0.75 & 0.2 & 0.05 & 0.75 & 0.2 & 0.05 & 0.9 & 0.1 & 0 & 0.9 & 0.1 & 0 \\
\hline F4 & 0.9 & 0.1 & 0 & 0.9 & 0.1 & 0 & 0.9 & 0.1 & 0 & 0.9 & 0.1 & 0 & 0.9 & 0.1 & 0 \\
\hline F5 & 0.9 & 0.1 & 0 & 0.75 & 0.2 & 0.05 & 0.75 & 0.2 & 0.05 & 0.75 & 0.2 & 0.05 & 0.9 & 0.1 & 0 \\
\hline F6 & 0.9 & 0.1 & 0 & 0.9 & 0.1 & 0 & 0.9 & 0.1 & 0 & 0.75 & 0.2 & 0.05 & 0.75 & 0.2 & 0.05 \\
\hline F7 & 0.9 & 0.1 & 0 & 0.9 & 0.1 & 0 & 0.9 & 0.1 & 0 & 0.9 & 0.1 & 0 & 0.9 & 0.1 & 0 \\
\hline F8 & 0.75 & 0.2 & 0.05 & 0.75 & 0.2 & 0.05 & 0.75 & 0.2 & 0.05 & 0.9 & 0.1 & 0 & 0.9 & 0.1 & 0 \\
\hline F9 & 0.9 & 0.1 & 0 & 0.75 & 0.2 & 0.05 & 0.75 & 0.2 & 0.05 & 0.75 & 0.2 & 0.05 & 0.75 & 0.2 & 0.05 \\
\hline F10 & 0.9 & 0.1 & 0 & 0.9 & 0.1 & 0 & 0.9 & 0.1 & 0 & 0.9 & 0.1 & 0 & 0.75 & 0.2 & 0.05 \\
\hline
\end{tabular}




\begin{tabular}{|c|c|c|c|c|c|c|c|c|c|c|c|c|c|c|c|c|}
\hline & \multicolumn{4}{|c|}{ F6 } & \multicolumn{4}{|c|}{ F7 } & \multicolumn{3}{c|}{ F8 } & \multicolumn{3}{c|}{ F9 } & \multicolumn{3}{c|}{ F10 } \\
\hline & $m(y)$ & $m(n)$ & $m(\theta)$ & $m(y)$ & $m(n)$ & $m(\theta)$ & $m(y)$ & $m(n)$ & $m(\theta)$ & $m(y)$ & $m(n)$ & $m(\theta)$ & $m(y)$ & $m(n)$ & $m(\theta)$ \\
\hline F1 & 0.9 & 0.1 & 0 & 0.75 & 0.2 & 0.05 & 0.9 & 0.1 & 0 & 0.75 & 0.2 & 0.05 & 0.75 & 0.2 & 0.05 \\
\hline F2 & 0.75 & 0.2 & 0.05 & 0.75 & 0.2 & 0.05 & 0.9 & 0.1 & 0 & 0.75 & 0.2 & 0.05 & 0.75 & 0.2 & 0.05 \\
\hline F3 & 0.9 & 0.1 & 0 & 0.75 & 0.2 & 0.05 & 0.75 & 0.2 & 0.05 & 0.75 & 0.2 & 0.05 & 0.75 & 0.2 & 0.05 \\
\hline F4 & 0.50 & 0.45 & 0.05 & 0.75 & 0.2 & 0.05 & 0.75 & 0.2 & 0.05 & 0.9 & 0.1 & 0 & 0.9 & 0.1 & 0.00 \\
\hline F5 & 0.50 & 0.45 & 0.05 & 0.75 & 0.2 & 0.05 & 0.9 & 0.1 & 0 & 0.9 & 0.1 & 0 & 0.9 & 0.1 & 0.00 \\
\hline F6 & 0.9 & 0.1 & 0 & 0.75 & 0.2 & 0.05 & 0.75 & 0.2 & 0.05 & 0.9 & 0.1 & 0 & 0.9 & 0.1 & 0.00 \\
\hline F7 & 0.9 & 0.1 & 0 & 0.9 & 0.1 & 0 & 0.75 & 0.2 & 0.05 & 0.50 & 0.45 & 0.05 & 0.50 & 0.45 & 0.05 \\
\hline F8 & 0.75 & 0.2 & 0.05 & 0.9 & 0.1 & 0 & 0.9 & 0.1 & 0 & 0.9 & 0.1 & 0 & 0.9 & 0.1 & 0.00 \\
\hline F9 & 0.75 & 0.2 & 0.05 & 0.75 & 0.2 & 0.05 & 0.75 & 0.2 & 0.05 & 0.9 & 0.1 & 0 & 0.9 & 0.1 & 0.00 \\
\hline F10 & 0.75 & 0.2 & 0.05 & 0.9 & 0.1 & 0 & 0.9 & 0.1 & 0 & 0.50 & 0.45 & 0.05 & 0.50 & 0.45 & 0.05 \\
\hline
\end{tabular}

Table 9: The value of BPA for the expert group 2(contd.)

\begin{tabular}{|c|c|c|c|c|c|c|c|c|c|c|c|c|c|c|c|}
\hline & \multicolumn{4}{|c|}{ F1 } & \multicolumn{4}{|c|}{ F2 } & \multicolumn{3}{c|}{ F3 } & \multicolumn{3}{c|}{ F4 } & \multicolumn{3}{c|}{ F5 } \\
\hline & $m(y)$ & $m(n)$ & $m(\theta)$ & $m(y)$ & $m(n)$ & $m(\theta)$ & $m(y)$ & $m(n)$ & $m(\theta)$ & $m(y)$ & $m(n)$ & $m(\theta)$ & $m(y)$ & $m(n)$ & $m(\theta)$ \\
\hline F1 & 0.75 & 0.2 & 0.05 & 0.9 & 0.1 & 0 & 0.9 & 0.1 & 0 & 0.75 & 0.2 & 0.05 & 0.75 & 0.2 & 0.05 \\
\hline F2 & 0.9 & 0.1 & 0 & 0.9 & 0.1 & 0 & 0.9 & 0.1 & 0 & 0.9 & 0.1 & 0 & 0.75 & 0.2 & 0.05 \\
\hline F3 & 0.75 & 0.2 & 0.05 & 0.35 & 0.60 & 0.05 & 0.35 & 0.60 & 0.05 & 0.9 & 0.1 & 0 & 0.9 & 0.1 & 0 \\
\hline F4 & 0.75 & 0.2 & 0.05 & 0.75 & 0.2 & 0.05 & 0.75 & 0.2 & 0.05 & 0.75 & 0.2 & 0.05 & 0.50 & 0.45 & 0.05 \\
\hline F5 & 0.75 & 0.2 & 0.05 & 0.75 & 0.2 & 0.05 & 0.75 & 0.2 & 0.05 & 0.35 & 0.60 & 0.05 & 0.50 & 0.45 & 0.05 \\
\hline F6 & 0.9 & 0.1 & 0 & 0.75 & 0.2 & 0.05 & 0.75 & 0.2 & 0.05 & 0.75 & 0.2 & 0.05 & 0.75 & 0.2 & 0.05 \\
\hline F7 & 0.9 & 0.1 & 0 & 0.9 & 0.1 & 0 & 0.9 & 0.1 & 0 & 0.75 & 0.2 & 0.05 & 0.75 & 0.2 & 0.05 \\
\hline F8 & 0.50 & 0.45 & 0.05 & 0.75 & 0.2 & 0.05 & 0.75 & 0.2 & 0.05 & 0.75 & 0.2 & 0.05 & 0.75 & 0.2 & 0.05 \\
\hline F9 & 0.75 & 0.2 & 0.05 & 0.50 & 0.45 & 0.05 & 0.50 & 0.45 & 0.05 & 0.50 & 0.45 & 0.05 & 0.75 & 0.2 & 0.05 \\
\hline F10 & 0.9 & 0.1 & 0 & 0.75 & 0.2 & 0.05 & 0.75 & 0.2 & 0.05 & 0.9 & 0.1 & 0 & 0.50 & 0.45 & 0.05 \\
\hline
\end{tabular}

Table 10: The value of BPA for the expert group-3

\begin{tabular}{|c|c|c|c|c|c|c|c|c|c|c|c|c|c|c|c|}
\hline & \multicolumn{3}{|c|}{ F6 } & \multicolumn{3}{|c|}{ F7 } & \multicolumn{3}{c|}{ F8 } & \multicolumn{3}{c|}{ F9 } & \multicolumn{3}{c|}{ F10 } \\
\hline & $m(y)$ & $m(n)$ & $m(\theta)$ & $m(y)$ & $m(n)$ & $m(\theta)$ & $m(y)$ & $m(n)$ & $m(\theta)$ & $m(y)$ & $m(n)$ & $m(\theta)$ & $m(y)$ & $m(n)$ & $m(\theta)$ \\
\hline F1 & 0.75 & 0.2 & 0.05 & 0.75 & 0.2 & 0.05 & 0.50 & 0.45 & 0.05 & 0.75 & 0.2 & 0.05 & 0.75 & 0.2 & 0.05 \\
\hline F2 & 0.75 & 0.2 & 0.05 & 0.50 & 0.45 & 0.05 & 0.75 & 0.2 & 0.05 & 0.75 & 0.2 & 0.05 & 0.75 & 0.2 & 0.05 \\
\hline F3 & 0.9 & 0.1 & 0 & 0.75 & 0.2 & 0.05 & 0.1 & 0.9 & 0 & 0.9 & 0.1 & 0 & 0.9 & 0.1 & 0.00 \\
\hline F4 & 0.9 & 0.1 & 0 & 0.75 & 0.2 & 0.05 & 0.75 & 0.2 & 0.05 & 0.75 & 0.2 & 0.05 & 0.75 & 0.2 & 0.05 \\
\hline F5 & 0.75 & 0.2 & 0.05 & 0.9 & 0.1 & 0 & 0.75 & 0.2 & 0.05 & 0.50 & 0.45 & 0.05 & 0.50 & 0.45 & 0.05 \\
\hline F6 & 0.50 & 0.45 & 0.05 & 0.50 & 0.45 & 0.05 & 0.75 & 0.2 & 0.05 & 0.75 & 0.2 & 0.05 & 0.75 & 0.2 & 0.05 \\
\hline F7 & 0.75 & 0.2 & 0.05 & 0.75 & 0.2 & 0.05 & 0.50 & 0.45 & 0.05 & 0.50 & 0.45 & 0.05 & 0.50 & 0.45 & 0.05 \\
\hline F8 & 0.50 & 0.45 & 0.05 & 0.50 & 0.45 & 0.05 & 0.50 & 0.45 & 0.05 & 0.75 & 0.2 & 0.05 & 0.75 & 0.2 & 0.05 \\
\hline F9 & 0.9 & 0.1 & 0 & 0.75 & 0.2 & 0.05 & 0.35 & 0.60 & 0.05 & 0.35 & 0.60 & 0.05 & 0.35 & 0.60 & 0.05 \\
\hline F10 & 0.9 & 0.1 & 0 & 0.75 & 0.2 & 0.05 & 0.75 & 0.2 & 0.05 & 0.9 & 0.1 & 0 & 0.9 & 0.1 & 0.00 \\
\hline
\end{tabular}

Table 11: The value of BPA for the expert group-3 (contd.) 
Step 3: Fuse the BPA matrix by adopting Dempster's rule of combination as given in Eq.(1) and Table-12 and Table-13. The each element of the BPA matrixes, a comprehensive matrix which denotes the initial relation matrix can be constructed for applying fuzzy DEMATEL approach.

\begin{tabular}{|c|c|c|c|c|c|c|c|c|c|c|}
\hline & F1 & F2 & F3 & F4 & F5 & F6 & F7 & F8 & F9 & F10 \\
\hline F1 & 0.000 & 0.729 & 0.729 & 0.576 & 0.648 & 0.648 & 0.576 & 0.446 & 0.576 & 0.576 \\
\hline F2 & 0.648 & 0.000 & 0.729 & 0.648 & 0.648 & 0.576 & 0.396 & 0.648 & 0.576 & 0.576 \\
\hline F3 & 0.576 & 0.288 & 0.000 & 0.729 & 0.729 & 0.729 & 0.512 & 0.072 & 0.648 & 0.648 \\
\hline F4 & 0.648 & 0.648 & 0.648 & 0.000 & 0.446 & 0.446 & 0.352 & 0.512 & 0.576 & 0.576 \\
\hline F5 & 0.648 & 0.576 & 0.576 & 0.288 & 0.000 & 0.396 & 0.648 & 0.648 & 0.446 & 0.446 \\
\hline F6 & 0.729 & 0.576 & 0.576 & 0.512 & 0.512 & 0.000 & 0.396 & 0.576 & 0.648 & 0.648 \\
\hline F7 & 0.729 & 0.648 & 0.729 & 0.576 & 0.648 & 0.648 & 0.000 & 0.396 & 0.272 & 0.272 \\
\hline F8 & 0.352 & 0.512 & 0.576 & 0.648 & 0.576 & 0.352 & 0.446 & 0.000 & 0.648 & 0.648 \\
\hline F9 & 0.648 & 0.352 & 0.396 & 0.396 & 0.576 & 0.648 & 0.512 & 0.288 & 0.000 & 0.324 \\
\hline F10 & 0.648 & 0.576 & 0.576 & 0.729 & 0.352 & 0.648 & 0.648 & 0.576 & 0.446 & 0.000 \\
\hline
\end{tabular}

Table 12: Results of fused BPA's for $m(y)$

\begin{tabular}{|c|c|c|c|c|c|c|c|c|c|c|}
\hline & F1 & F2 & F3 & F4 & F5 & F6 & F7 & F8 & F9 & F10 \\
\hline F1 & 0.000 & 0.001 & 0.001 & 0.006 & 0.003 & 0.003 & 0.006 & 0.005 & 0.006 & 0.006 \\
\hline F2 & 0.003 & 0.000 & 0.001 & 0.003 & 0.003 & 0.006 & 0.013 & 0.003 & 0.006 & 0.006 \\
\hline F3 & 0.006 & 0.016 & 0.000 & 0.001 & 0.001 & 0.001 & 0.016 & 0.023 & 0.003 & 0.003 \\
\hline F4 & 0.003 & 0.003 & 0.003 & 0.000 & 0.005 & 0.005 & 0.031 & 0.016 & 0.006 & 0.006 \\
\hline F5 & 0.003 & 0.006 & 0.006 & 0.016 & 0.000 & 0.013 & 0.003 & 0.003 & 0.005 & 0.005 \\
\hline F6 & 0.001 & 0.006 & 0.006 & 0.016 & 0.016 & 0.000 & 0.013 & 0.006 & 0.003 & 0.003 \\
\hline F7 & 0.001 & 0.003 & 0.001 & 0.006 & 0.003 & 0.003 & 0.000 & 0.013 & 0.025 & 0.025 \\
\hline F8 & 0.031 & 0.016 & 0.006 & 0.003 & 0.006 & 0.031 & 0.005 & 0.000 & 0.003 & 0.003 \\
\hline F9 & 0.003 & 0.031 & 0.013 & 0.013 & 0.006 & 0.003 & 0.016 & 0.016 & 0.000 & 0.007 \\
\hline F10 & 0.003 & 0.006 & 0.006 & 0.001 & 0.031 & 0.003 & 0.003 & 0.006 & 0.005 & 0.000 \\
\hline
\end{tabular}

Table 13: Results of fused BPA's for $\boldsymbol{m}(\boldsymbol{n})$

\subsection{Evaluation of KT effectiveness criteria's by Fuzzy DEMATEL}

The basic steps of fuzzy DEMATEL approach used in this study are as follows:

Step 1: Convert the fuzzy linguistic assessments into crisp scores. Set up the initial direct relational matrix.

Step 2: Construct the generalized direct relational matrix and total relation matrix to obtain weights of the criteria as shown in Table 14 and Table-15. 
Step 3: Obtain Prominence and relation value and compute the importance of the criteria as shown in Table-16 and Table-17.

\begin{tabular}{|c|c|c|c|c|c|c|c|c|c|c|}
\hline & F1 & F2 & F3 & F4 & F5 & F6 & F7 & F8 & F9 & F10 \\
\hline F1 & 1.043 & 1.033 & 1.142 & 1.043 & 1.064 & 1.060 & 0.941 & 0.858 & 1.004 & 0.983 \\
\hline F2 & 1.133 & 0.903 & 1.128 & 1.042 & 1.052 & 1.036 & 0.903 & 0.878 & 0.993 & 0.973 \\
\hline F3 & 1.037 & 0.877 & 0.921 & 0.968 & 0.979 & 0.978 & 0.848 & 0.724 & 0.923 & 0.904 \\
\hline F4 & 1.034 & 0.922 & 1.019 & 0.847 & 0.930 & 0.927 & 0.815 & 0.783 & 0.907 & 0.888 \\
\hline F5 & 1.002 & 0.884 & 0.979 & 0.869 & 0.828 & 0.890 & 0.836 & 0.780 & 0.858 & 0.840 \\
\hline F6 & 1.101 & 0.960 & 1.061 & 0.981 & 0.990 & 0.901 & 0.867 & 0.834 & 0.965 & 0.945 \\
\hline F7 & 1.066 & 0.939 & 1.051 & 0.957 & 0.979 & 0.973 & 0.769 & 0.780 & 0.876 & 0.858 \\
\hline F8 & 0.964 & 0.878 & 0.981 & 0.927 & 0.924 & 0.888 & 0.809 & 0.679 & 0.893 & 0.875 \\
\hline F9 & 0.911 & 0.768 & 0.858 & 0.796 & 0.834 & 0.842 & 0.738 & 0.655 & 0.700 & 0.740 \\
\hline F10 & 1.098 & 0.969 & 1.072 & 1.023 & 0.974 & 1.015 & 0.910 & 0.841 & 0.941 & 0.847 \\
\hline
\end{tabular}

Table 14: Total relation matrix according to $m(y)$

\begin{tabular}{|r|c|c|c|c|c|c|c|c|c|c|}
\hline & F1 & F2 & F3 & F4 & F5 & F6 & F7 & F8 & F9 & F10 \\
\hline F1 & 0.062 & 0.107 & 0.060 & 0.128 & 0.109 & 0.102 & 0.166 & 0.141 & 0.134 & 0.136 \\
\hline F2 & 0.084 & 0.106 & 0.065 & 0.106 & 0.120 & 0.137 & 0.227 & 0.129 & 0.151 & 0.153 \\
\hline F3 & 0.191 & 0.302 & 0.077 & 0.121 & 0.137 & 0.171 & 0.307 & 0.346 & 0.160 & 0.163 \\
\hline F4 & 0.159 & 0.217 & 0.122 & 0.146 & 0.212 & 0.216 & 0.476 & 0.331 & 0.239 & 0.243 \\
\hline F5 & 0.106 & 0.190 & 0.127 & 0.252 & 0.122 & 0.221 & 0.208 & 0.170 & 0.154 & 0.156 \\
\hline F6 & 0.112 & 0.209 & 0.137 & 0.265 & 0.267 & 0.140 & 0.308 & 0.223 & 0.163 & 0.165 \\
\hline F7 & 0.145 & 0.254 & 0.128 & 0.205 & 0.226 & 0.193 & 0.222 & 0.309 & 0.367 & 0.372 \\
\hline F8 & 0.392 & 0.317 & 0.156 & 0.199 & 0.233 & 0.431 & 0.285 & 0.192 & 0.182 & 0.185 \\
\hline F9 & 0.188 & 0.502 & 0.224 & 0.273 & 0.244 & 0.232 & 0.415 & 0.376 & 0.198 & 0.259 \\
\hline F10 & 0.112 & 0.197 & 0.133 & 0.137 & 0.386 & 0.155 & 0.173 & 0.185 & 0.149 & 0.106 \\
\hline
\end{tabular}

Table 15: Total relation matrix according to $m(n)$

\begin{tabular}{|c|c|c|c|c|c|}
\hline Priority & $\mathbf{R}$ & Priority & $\mathbf{C}$ & Priority & R-C \\
\hline F1 & 10.17 & F1 & 10.388 & F10 & -0.218 \\
\hline F2 & 10.04 & F2 & 9.345 & F8 & 0.696 \\
\hline F10 & 9.69 & F3 & 8.212 & F9 & 1.477 \\
\hline F6 & 9.61 & $\mathrm{~F} 4$ & 7.176 & F7 & 2.430 \\
\hline F7 & 9.25 & F5 & 6.203 & F6 & 3.045 \\
\hline F3 & 9.16 & F6 & 5.285 & F5 & 3.874 \\
\hline F4 & 9.07 & F7 & 4.375 & F4 & 4.696 \\
\hline F8 & 8.82 & F8 & 3.468 & F3 & 5.350 \\
\hline F5 & 8.77 & F9 & 2.610 & F2 & 6.156 \\
\hline F9 & 7.84 & F10 & 1.812 & F1 & 6.031 \\
\hline
\end{tabular}

Table 16: Level of influence of factors according to $\boldsymbol{m}(\boldsymbol{y})$

\begin{tabular}{|c|c|c|c|c|c|}
\hline Priority & R & Priority & C & Priority & R-C \\
\hline F9 & 2.912 & F1 & 1.551 & F9 & 1.362 \\
\hline F8 & 2.572 & F2 & 1.489 & F8 & 1.083 \\
\hline F7 & 2.423 & F3 & 1.405 & F10 & 1.018 \\
\hline F4 & 2.362 & F4 & 1.214 & F7 & 1.147 \\
\hline F6 & 1.988 & F5 & 1.055 & F4 & 0.933 \\
\hline F3 & 1.976 & F6 & 0.949 & F6 & 1.027 \\
\hline F10 & 1.733 & F7 & 0.837 & F5 & 0.896 \\
\hline
\end{tabular}




\begin{tabular}{|l|c|c|c|c|c|} 
F5 & 1.706 & F8 & 0.692 & F3 & 1.014 \\
\hline F2 & 1.277 & F9 & 0.300 & F1 & 0.978 \\
\hline F1 & 1.144 & F10 & 0.112 & F2 & 1.032 \\
\hline
\end{tabular}

Table 17: Level of influence of factors according to $m(n)$

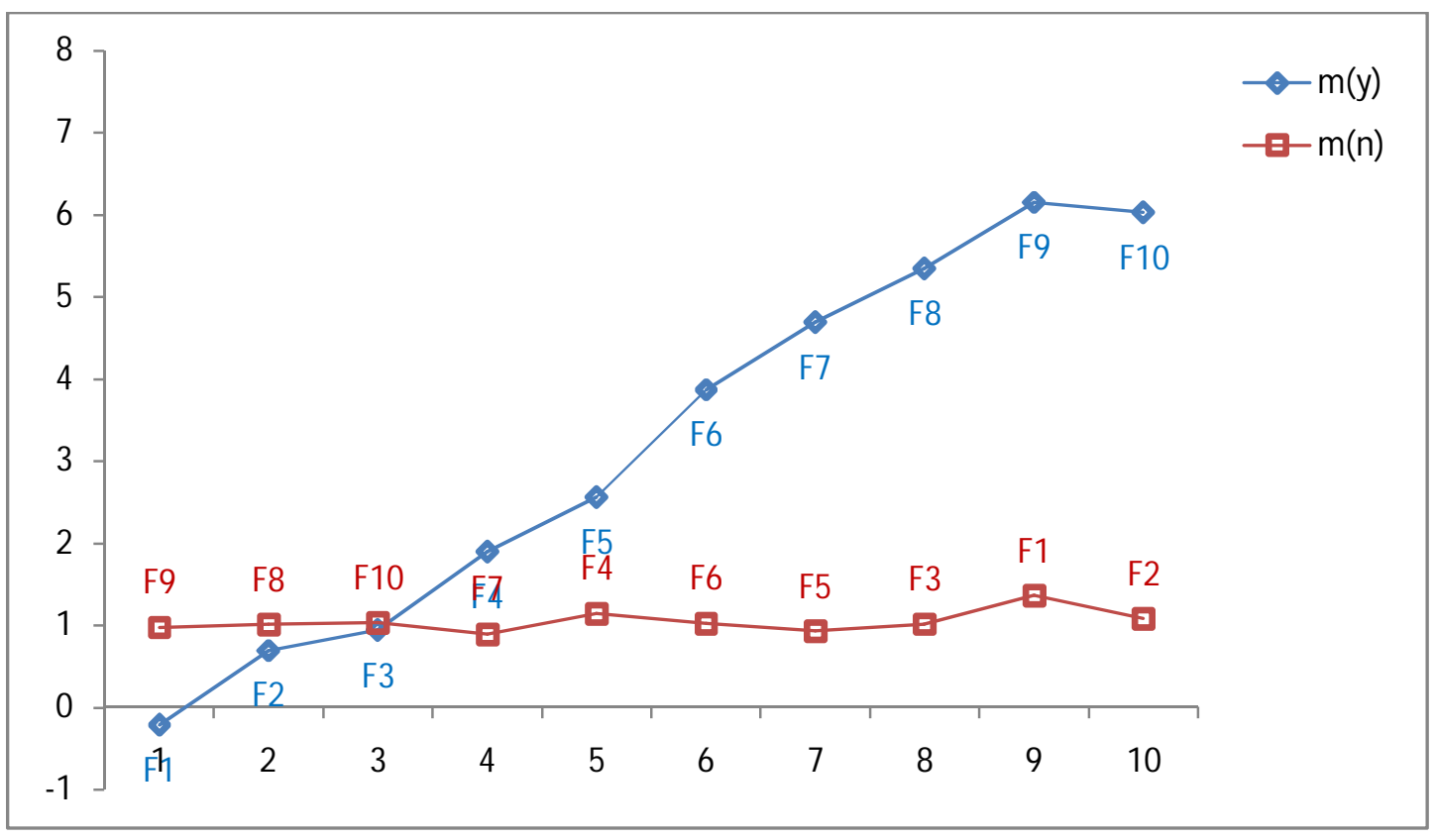

Figure 2: Prioritization of factors with respect to KT effectiveness of GSD teams

\section{Results and Discussion}

In relation to the four main contexts and ten evaluation criteria for the possible ratings of KT effectiveness with reference to GSD projects has summarized in Table-1. The expert decision groups of this study were executive committee, solution development team, and solution delivery team of Inowits software organization to evaluate the GSD teams KT effectiveness criteria using IFNs. As a result, three initial direct relational matrixes have been formed as shown in table-3 to table-5. In addition, table- 6 to table-11 shows the IFNs to BPA matrixes conversion with respect to the criteria for each criterion. Subsequently, the Dempster-Shafer theory is used to fuse the BPA matrices of three expert matrices into a single comprehensive matrix in which elements are $\{m(y), m(n), m(\theta)\}$ as given in table-12 and table-13. The initial direct relation matrix constructed thus it is used in the fuzzy DEMATEL approach. Once the total relation matrix has been computed for the membership and non-membership functions as shown in table-14 and Table-15, the level of influence, and level of relationships have been obtained. 
The indexes R, C, and R-C have been computed via table-14 and 15 respectively. Following the prioritization factors influencing KT effectiveness of GSD teams are figured out as depicted in Fig.2. In relation to the four main contexts and ten evaluation criteria for the possible ratings of KT effectiveness with reference to GSD projects has summarized in table-16 and table-17. For each factor $\mathrm{R}-\mathrm{C}$ is calculated in which the results address that factors having greater values of $\mathrm{R}$ - $\mathrm{C}$ have higher impact to another and it's assumed to have higher rank. So, $\mathrm{R}-\mathrm{C}$ is a good criterion for factors ranking. Therefore, factors having greater values of $\mathrm{R}-\mathrm{C}$ on $m(y)$ have higher influence compare to other factors and it denotes the higher priority. On other hand, factors having lower values of $\mathrm{R}-\mathrm{C}$ on $m(n)$ have higher influence compare to other factors and it denotes the higher priority. In summary the values of $\mathrm{R}-\mathrm{C}$ on $m(y)$ and $m(n)$, we can obtain a comprehensive final result in level of influential factors.

The results address that $\mathrm{F} 8, \mathrm{~F} 9, \mathrm{~F} 10$ have higher values of $m(y)$ and $\mathrm{F} 3, \mathrm{~F} 1, \mathrm{~F} 2$ have higher values of $m(n)$ when compare to other values. To aggregate the row values of total relational matrix in table-14, first row values are higher compare to other row values according to $m(y)$. similarly, to aggregate the row values of total relational matrix in Table-15, third row values are greater than other row values according to $m(n)$. while the highest values of Table-14 greater impact and lower value in Table-15. Thus, the factor F9 has highest influential factor compare to all other factors. In this context, F9 can be most influential factor to address the KT effectiveness of GSD teams. Likewise, factor F7, F6, F10 and F8 also reveals the significant impact on KT effectiveness. Based on this result, the factors are divided into two categories: Most Significant Factors (MSFs - F7, F9, F6, F10 and F8) and Least Significant Factors (LSFs - F1, F3, F4, F2 and F5). From the first row of Table 14, we aggregate the values among MSFs is lower values than the LSFs. The computation process is given below:

MSF: $0.738+0.700+0.842+0.740+0.655=3.675$

LSF: $0.911+0.858+0.796+0.768+0.834=4.167$

Sum of the values among MSFs is also lower than the values of LSFs. These results addressed that MSFs have higher impact than LSFs, which denotes that if the performance of MSFs is improved, the performance of LSFs can be greatly acknowledged for KT effectiveness in GSD projects. 


\section{Conclusion}

In the context of GSD teams' organizational behavior research phenomenon, this research presents a framework to evaluate the KT effectiveness in GSD projects which integrates two aspects: (i) Dempster-Shafer theory and (ii) fuzzy DEMATEL. Moreover, this integrated approach has been investigated in Inowits software organization to explore the significance of the KT effectiveness of offshore/onsite teams in GSD projects. The results clearly indicates that GSD teams KT effectiveness factors reveals key determinant to achieve the GSD project outcome, while their priority relationship and its measures across the criteria under fuzzy environment presented in this paper.

Consequently, this study has presents two valuable contributions: (i) a comprehensive framework of the factors influencing KT effectiveness of GSD teams (ii) Dempster-Shafer theory DEMATEL approach to find the relative importance of the criteria and to priority the criteria on the basis of intuitionistic fuzzy information. In addition, uncertainty and subjective vagueness within the decision making process, has been effectively handled through IFNs. The proposed approach can be more helpful industry for evaluating KT effectiveness of GSD teams. Similarly, the case study results of this research provide a vivid picture the significance of OB research on offshore/onsite teams' which addresses the significance of KT evaluation criteria's for the outcome of GSD projects. In future, we could include more criteria for KT effectiveness of GSD teams and integrating other fuzzy MCDM approaches to fuse in intuitionistic fuzzy information that is focused in subsequent studies.

\section{References}

[1] Arun Kumar Sangaiah, and Thangavelu, A.K. (2013). An adaptive neuro-fuzzy approach to evaluation of team level service climate in GSD projects, Neural Computing and Application, Springer Publishers, Vol.25(3-4),573-583.(DOI: 10.1007/s00521-013-15219)

[2] Arun Kumar, S., Thangavelu, Arun Kumar (2013). Factors affecting the outcome of Global Software Development projects: An empirical study, International Conference on Computer Communication and Informatics (ICCCI), IEEE Explore, 1-10, doi: 10.1109/ICCCI.2013.6466113. 
[3] Arun Kumar. S. and Arun Kumar Thangavelu (2012). Exploring the influence of partnership quality factors towards the outcome of Global Software Development Projects, International review on computer and software, Vol.7, No.5, pp.2159-2172.

[4] Sangaiah, A. K., \& Thangavelu, A. K.: An exploration of FMCDM approach for evaluating the outcome/success of GSD projects. Central European Journal of Engineering, 3(3), 419-435 (2013).

[5] Sangaiah, A. K., Subramaniam, P. R., \& Zheng, X. A. (2014) combined fuzzy DEMATEL and fuzzy TOPSIS approach for evaluating GSD project outcome factors. Neural Computing and Applications, Article in Press (DOI: 10.1007/s00521-014-1771-1)

[6] Gopal, J., Sangaiah, A.K., Basu, A., Pradeep Reddy, C. (2015) Towards identifying the knowledge codification effects on the factors affecting knowledge transfer effectiveness in the context of GSD project outcome. Advances in Intelligent Systems and Computing, 337, pp. 611-620.

[7] Gopal, J., Sangaiah, A.K., Basu, A., Gao, X.Z. (2015) Integrating Fuzzy DEMATEL and FMCDM Approach for Evaluating Knowledge Transfer Effectiveness with Reference to GSD project Outcome. Manuscript submitted to International Journal of Machine Learning and Cybernatics.

[8] Li, Y., Hu, Y., Zhang, X., Deng, Y., \& Mahadevan, S. (2014). An evidential DEMATEL method to identify critical success factors in emergency management. Applied Soft Computing. 22, pp. 504-510.

[9] Patil, S. K., \& Kant, R. (2014). A hybrid approach based on fuzzy DEMATEL and FMCDM to predict success of knowledge management adoption in supply chain. Applied Soft Computing, 18, 126-135.

[10] LI, Y., SHU, G., DENG, X., \& DENG, Y. (2014). A Multi-attribute Decision Making Method Based on Evidence Theory and Average Operator*. Journal of Computational Information Systems, 10(2), 595-601.

[11] Shafer, G. (1976). A mathematical theory of evidence (Vol. 1). Princeton: Princeton university press.

[12] Y. Deng, F. Chan, (2011). A new fuzzy Dempster MCDM method and its application in supplier selection, Expert Syst. Appl. 38 (8) 6985-6993.

[13] Xinyang Deng, Xi Zheng, Xiaoyan Su, Felix T.S. Chan, Yong Hu, Rehan Sadiq, Yong Deng,(2014). An evidential game theory framework in multi-criteria decision making process, Applied Mathematics and Computation, 244, pp.783-793.

[14] Z. Xu., Yager., R.R, (2006) Some geometric aggregation operators based on intuitionistic fuzzy sets, Int. J. General Syst. 35 (4) 417-433. 
[15] Atanassov, K. T. (1986). Intuitionistic fuzzy sets. Fuzzy sets and Systems, 20(1), 87-96.

[16] Boran, F. E., Genç, S., Kurt, M., \& Akay, D. (2009). A multi-criteria intuitionistic fuzzy group decision making for supplier selection with TOPSIS method. Expert Systems with Applications, 36(8), 11363-11368.

[17] Sevastianov, P. (2007). Numerical methods for interval and fuzzy number comparison based on the probabilistic approach and Dempster-Shafer theory. Information Sciences, 177(21), 4645-4661.

[18] Arun Kumar, S., Thangavelu, A. K., \& Ashok Kumar, P. M. (2014). Partnership quality and service climate aspects in the context of GSD project outcome- a case study. Int $J$ Appl Eng Res, 9(16), 3203-3223.

[19] Sangaiah, A. K., \& Thangavelu, A. K. (2013). Measuring IT service quality in the context of team-level service climate and GSD project outcome relationship. Australian Journal of Basic \& Applied Sciences, 7(8).

[20] Yager, R. R. (1987). On the Dempster-Shafer framework and new combination rules. Information sciences, 41(2), 93-137. 